OPEN ACCESS

Edited by:

Lavinia Alberi,

University of Fribourg, Switzerland

Reviewed by:

Adelaide Fernandes,

University of Lisbon, Portugal

Stefania Ceruti,

Università degli Studi di Milano, Italy

${ }^{*}$ Correspondence:

Servio H. Ramirez

servio.ramirez@temple.edu

Received: 19 December 2015 Accepted: 08 February 2016

Published: 29 February 2016

Citation:

Andrews AM, Lutton EM, Merkel SF, Razmpour R and Ramirez SH (2016)

Mechanical Injury Induces Brain Endothelial-Derived Microvesicle Release: Implications for Cerebral Vascular Injury during Traumatic Brain Injury. Front. Cell. Neurosci. 10:43. doi: 10.3389/fncel.2016.00043

\section{Mechanical Injury Induces Brain Endothelial-Derived Microvesicle Release: Implications for Cerebral Vascular Injury during Traumatic Brain Injury}

\author{
Allison M. Andrews ${ }^{1,2}$, Evan M. Lutton ${ }^{1}$, Steven F. Merkel ${ }^{1,2}$, Roshanak Razmpour ${ }^{1}$ and \\ Servio H. Ramirez ${ }^{1,2,3 *}$ \\ ${ }^{1}$ Department of Pathology and Laboratory Medicine, Lewis Katz School of Medicine at Temple University, Philadelphia, PA, \\ USA, ${ }^{2}$ The Shriners Hospitals Pediatric Research Center, Philadelphia, PA, USA, ${ }^{3}$ The Center for Substance Abuse \\ Research, Lewis Katz School of Medicine at Temple University, Philadelphia, PA, USA
}

It is well established that the endothelium responds to mechanical forces induced by changes in shear stress and strain. However, our understanding of vascular remodeling following traumatic brain injury (TBI) remains incomplete. Recently published studies have revealed that lung and umbilical endothelial cells produce extracellular microvesicles (eMVs), such as microparticles, in response to changes in mechanical forces (blood flow and mechanical injury). Yet, to date, no studies have shown whether brain endothelial cells produce eMVs following TBI. The brain endothelium is highly specialized and forms the blood-brain barrier (BBB), which regulates diffusion and transport of solutes into the brain. This specialization is largely due to the presence of tight junction proteins (TJPs) between neighboring endothelial cells. Following TBI, a breakdown in tight junction complexes at the BBB leads to increased permeability, which greatly contributes to the secondary phase of injury. We have therefore tested the hypothesis that brain endothelium responds to mechanical injury, by producing eMVs that contain brain endothelial proteins, specifically TJPs. In our study, primary human adult brain microvascular endothelial cells (BMVEC) were subjected to rapid mechanical injury to simulate the abrupt endothelial disruption that can occur in the primary injury phase of TBI. eMVs were isolated from the media following injury at 2, 6, 24, and $48 \mathrm{~h}$. Western blot analysis of eMVs demonstrated a time-dependent increase in TJP occludin, PECAM-1 and ICAM-1 following mechanical injury. In addition, activation of ARF6, a small GTPase linked to extracellular vesicle production, was increased after injury. To confirm these results in vivo, mice were subjected to sham surgery or TBI and blood plasma was collected $24 \mathrm{~h}$ post-injury. Isolation and analysis of eMVs from blood plasma using cryo-EM and flow cytometry revealed elevated levels of vesicles containing occludin following brain trauma. These results indicate that following TBI, the cerebral endothelium undergoes vascular remodeling through shedding of eMVs containing TJPs and endothelial markers. The detection of this shedding potentially allows for a novel 
methodology for real-time monitoring of cerebral vascular health (remodeling), BBB status and neuroinflammation following a TBI event.

Keywords: blood-brain barrier (BBB), traumatic brain injury, mechanical strain, extracellular microvesicles, tight junction proteins, neuroinflammation

\section{INTRODUCTION}

TBI represents a serious medical concern for patients and has both short-term (disorientation, memory loss etc.) and long-term (disability, loss of productivity etc.) effects. The pathology of TBI is due to a combination of primary (mechanical) and subsequent secondary (biochemical and cellular) effects. While previous TBI research has focused on damage to neurons, recent evidence has highlighted the central role of vascular integrity and changes in BBB permeability in delayed neuronal death and dysfunction (Shlosberg et al., 2010). In some cases, BBB dysfunction can last for months or years following the original injury (Korn et al., 2005). Prolonged BBB permeability can contribute to edema formation and result in cytotoxicity and further neuronal damage (Unterberg et al., 2004). Such observations are not only limited to cases of moderate or severe TBI, but can also be seen within the mild spectrum of TBI, including concussive and sub-concussive injuries. Part of its pathologic description commonly mentions the lack of radiological presentation of a hematoma, however this does not eliminate the possibility that the BBB has not been compromised. In fact, recent findings in mild TBI research have revealed the existence of micro-tears, which are small gaps or separations observed between endothelial cells, and sites of BBB breach (Glushakova et al., 2014; Liu et al., 2016). Thus, all forms of TBI at both the primary phase (induced by forces that are linear or rotational) and the secondary phase (a result of inflammation) trigger changes to the $\mathrm{BBB}$, particularly at the level of single cell thick capillaries.

The brain endothelium is unique from other endothelium in that it has highly enriched levels of tight junction proteins (TJPs) such as occludin, claudin (Cld $-3,-5$ and -12 ), zonula occludens proteins $(\mathrm{ZO}-1,-2)$, and junctional adhesion molecules (JAM; Abbott et al., 2006). BBB integrity and formation of tight junctions (TJ) between endothelial cells (ECs) are vital for proper barrier function. However, our understanding of how mechanical injury and the secondary biochemical changes that occur in TBI affect TJs and TJPs remains unclear.

It is thought that all or most cells produce extracellular microvesicles (eMVs), which include microparticles and exosomes, in response to stimuli, activation, and injury. The release of eMVs has been shown to result from upstream signaling involving cytoskeletal remodeling and activation of GTPases RhoA and ARF6 (Boshans et al., 2000; MuralidharanChari et al., 2009; Latham et al., 2013; Ghossoub et al., 2014). Previous reports have shown that lung and umbilical ECs produce microparticles in response to mechanical stimuli (physiological shear stress and cyclic strain) in vitro (Vion et al., 2013a,b; Letsiou et al., 2015) and in vivo (Jenkins et al., 2013). In addition, an immortalized brain endothelial cell line (hCMEC/D3) has been shown to produce microparticles in response to cytokine and agonist stimulation (Latham et al.,
2013). However, to date, no studies have shown whether brain ECs produce eMVs following TBI or whether eMVs contain TJPs that may reflect changes in BBB integrity. A recent study has shown elevated levels of the TJP occludin in the serum following a mild TBI (Shan et al., 2016); however, the eMV protein expression profile was never investigated.

To test whether TBI induces the release of brain endothelialderived eMVs, we utilized an in vitro model that simulates tissue deformation caused by rapid changes in linear strain (such as that produced in TBI) applied to human brain endothelial cells (BMVECs). The model consists of BMVECs grown on elastic membranes that are deformed by vacuum to deliver a rapid injury. Our results indicate that the injury induces a time-dependent release of eMVs containing the TJP occludin. In addition, we detected elevated levels of endothelial markers in eMVs and increased endothelial activation of the small GTPase protein ARF6. Next, we confirmed the elevated release of occludin in vivo using the controlled cortical impact model of TBI. In addition, cryo-EM and immune-gold labeling of occludin demonstrated localization of occludin in eMVs from blood plasma following a TBI event. Overall, these results suggest that TJPs from cells that form the BBB are readily produced and detectable following mechanical injury to the endothelium. Importantly these findings also reveal the potential for using brain endothelial-derived eMVs as biosignatures for monitoring the health of the $\mathrm{BBB}$ not only during brain injury but possibly for other neurological conditions as well.

\section{MATERIALS AND METHODS}

\section{Reagents}

Rat-tail collagen I and endothelial cell growth supplement (ECGS) were purchased from BD Biosciences (Franklin Lakes, NJ). Heparin and bovine serum albumin (Sigma), DMEM/Ham's F12 media, Fetal bovine serum (FBS; Thermo Fisher Scientific, Waltham, MA).

\section{Endothelial Cell Culture}

Primary human adult brain microvascular endothelial cells (BMVECs) were provided by Michael Bernas and Dr. Marlys Witte (University of Arizona, Tucson). BMVECs were isolated from vessels from the brain resection path of patients (showing no abnormalities) undergoing surgery for the treatment of intractable epilepsy, as previously described (Bernas et al., 2010). Isolation of cells and tissue and the use of BMVECs has been approved by Temple University and the University of Arizona's IRB committees. BMVECs were grown in Dulbecco's modified Eagle's medium/F12 media supplemented with $10 \%$ heatinactivated fetal bovine serum (FBS; Thermo Fisher Scientific), endothelial cell growth supplement (ECGS; BD Bioscience), heparin $(1 \mathrm{mg} / \mathrm{mL}$; Sigma/Aldrich Co, Ltd), amphotericin B 
$(2.5 \mu \mathrm{g} / \mathrm{mL}$; Thermo Fisher Scientific), penicillin $(100 \mathrm{U} / \mathrm{mL}$; Thermo Fisher Scientific), and streptomycin $(100 \mu \mathrm{g} / \mathrm{mL}$; Thermo Fisher Scientific).

\section{In vitro Mechanical Strain-Induced Injury Model}

BMVECs were plated on Collagen I coated 6-well Flexcell plates (Flexcell International Corporation) and grown until confluence. The day before all experiments, media was exchanged with $10 \%$ FBS in DMEM F12 and then all experiments were conducted in 1\% FBS DMEM F12. Endothelial cells were rapidly injured using a $1 \mathrm{~s}$ gradient biaxial strain ( 12 or $22 \%$ substrate elongation) exerted by vacuum application to the chamber using the Flexcell Tension System FX-5000 (Flexcell International Corporation). Percentage of Elongation is defined by the following equation:

$$
\% \text { Elongation }=\frac{L_{\text {final }}-L_{\text {initial }}}{L_{\text {initial }}} * 100
$$

Where $L$ is the length of the substrate. The elongation of the membrane is a function of the applied force (from the vacuum) and the elasticity of the membrane. The following equation describes the force applied based on the vacuum pressure exerted:

$$
F=0.177 * P * D^{2}
$$

Where $F$ is the force applied in lbs, $P$ is the pressure in $\mathrm{MPa}$ and $D$ is the diameter of the sample in $\mathrm{mm}$. The required force to achieve the desired \% elongation is pre-programed by the manufacturer based on specifications for the Flexcell plates. Following injury, cells and media were harvested at time points ( 5 min and 2, 6, 24, 48 h) and processed for further analysis. For eMV harvest, media was spun at $2000 \times \mathrm{g}$ for $20 \mathrm{~min}$ to remove cellular debris and the supernatant was snap frozen and stored at $-80^{\circ} \mathrm{C}$. ARF6 activation was determined using a G-LISA assay (Cytoskeleton Inc., Denver, CO) following the manufacturer's protocol. A constitutively active ARF6 protein provided with the assay was used as a positive control.

\section{Live Cell Imaging and Immunocytochemistry}

For visualization of live cells after injury, cells were incubated with $1 \mu \mathrm{M}$ of the cell tracker dye, Calcein-AM (Thermo Fisher Scientific) for $20 \mathrm{~min}$ prior to injury. Images were taken prior to and $2 \mathrm{~h}$ post injury. For immunocytochemistry, cells were fixed with $4 \%$ paraformaldehyde at $2 \mathrm{~h}$ post injury. The cells were then permeabilized with $0.1 \%$ triton for $10 \mathrm{~min}$ and then non-specific binding was blocked using 5\% BSA for $30 \mathrm{~min}$. Incubation of cells with primary antibodies to ZO-1 (BD Biosciences) was then performed overnight at $4^{\circ} \mathrm{C}$. After rinsing at least three times with $1 \mathrm{x}$ PBS the cells were incubated with species specific Alexa488 conjugated secondary antibodies for $1 \mathrm{~h}$ at RT (Thermo Fisher Scientific), rhodamine conjugated phalloidin for $1 \mathrm{~h}$ at RT (Cytoskeleton, Inc.) followed by counterstaining with nuclear dye DAPI for $30 \mathrm{~min}$. Cells were then rinsed three times in 1x PBS and mounted in Prolong Antifade (Thermo Fisher Scientific) prior to coverslipping. All images were captured using a Coolsnap EZ
CCD camera (Photometrics, Tucson, AZ) coupled to a Nikon 80i Eclipse (Nikon, Japan) and processed using the NIS Elements imaging software (Nikon).

\section{Flow Cytometry of BMVECs}

Metabolic activity assay: metabolic activity was determined using the dye $\mathrm{C}_{12}$-resazurin, which converts to red-fluorescent $\mathrm{C}_{12}$-resorufin in metabolically active cells (Thermo Fisher Scientific). Cells were rinsed with $1 \mathrm{x}$ PBS and then trypsinized. $\mathrm{C}_{12}$-resazurin was added (to a final concentration of $500 \mathrm{nM}$ ) and cells were incubated for $15 \mathrm{~min}$ at $37^{\circ} \mathrm{C}$. Cells were washed and placed on ice and the fluorescence output was measured immediately. Cell Death Assay: \% of dead cells was determined using STOX ${ }^{\circledR}$ Green, a green-fluorescent nuclear and chromosome counterstain that is impermeant to live cells (Thermo Fisher Scientific). Detached cells were pelleted from the media and combined with adherent cells, which were removed by trypsinization. STOX ${ }^{\circledR}$ Green was added to a final concentration of $10 \mathrm{nM}$ and cells were incubated for $15 \mathrm{~min}$ at $37^{\circ} \mathrm{C}$. Cells were then washed, placed on ice and the fluorescence output was measured immediately. Surface expression immunostaining of ICAM-1: Cells were rinsed with calcium and magnesium free 1x PBS, trypsinized and then pelleted by centrifugation at $1000 \mathrm{rpm}$ for $5 \mathrm{~min}$. Cells were then resuspended in fixation buffer (eBioscience Inc., San Diego, CA) and incubated for $10 \mathrm{~min}$. Following fixation, cells were washed with flow cytometry buffer (eBioscience) and centrifuged as above. Cells were resuspended in $100 \mu \mathrm{L}$ of flow cytometry buffer with an ICAM-1 preconjugated antibody to R-phycoerythrin (PE; eBioscience) for $1 \mathrm{~h}$. Cells were then rinsed, centrifuged and resuspended in flow cytometry buffer for flow cytometry analysis. Data was acquired with a FACS BD Canto II flow cytometer (BD Biosciences) and analyzed using the FlowJo software (Tree Star, Ashland, OR, USA).

\section{Western Blot of eMVs}

eMVs were isolated using the Exoquick-TC system from System Biosciences Inc. (Mountain View, CA) following the manufacturer recommendation. eMVs were isolated from equal media volumes produced from equal cell numbers in order to control for differences in quantities of eMVs produced between conditions. All cell counts were obtained using an automated cell counter (Scepter, EMD Millipore, Billerica MA). The eMVs were then centrifuged and resuspended in Cell Lytic MT Cell Lysis Reagent (Sigma). Lysed eMVs were then mixed with $4 \mathrm{x}$ Laemmli buffer and boiled for $5 \mathrm{~min}$ at $95^{\circ} \mathrm{C}$. Samples were loaded onto a 4-20\% Mini-Protean TGX gel (Biorad, Hercules, CA). Gels were transferred to nitrocellulose membranes using the Trans-blot Turbo ${ }^{\mathrm{TM}}$ transfer system (Biorad) following the manufacturer's protocol. Membranes were blocked with 5\% nonfat dry milk in PBST (0.5\%) and all primary and secondary antibodies were resuspended in $5 \%$ nonfat dry milk in PBST $(0.5 \%)$. Primary and secondary antibodies used were as follows: occludin (abcam, Cambridge, UK, rabbit, 1:1000), PECAM-1 (Santa Cruz Biotechnology Inc., Santa Cruz, CA, goat 1:1000), ICAM (Santa Cruz Biotechnology Inc., rabbit 1:1000), goat-HRP (Jackson ImmunoResearch 
1:10,000) rabbit-HRP (GE Healthcare, Princeton, NJ, 1:10,000). HRP-conjugated antibodies were detected using Supersignal West Pico chemiluminescent substrate (Thermo Fisher Scientific) and visualization of luminescent signal was obtained using the gel documentation system, G:Box Chemi HR16 (Syngene, Frederick, MD). Densitometry was performed with the image analysis software, Image J $1.48 \mathrm{v}(\mathrm{NIH})$

\section{Controlled Cortical Impact (CCl) Mouse Model of Traumatic Brain Injury}

The Institutional Animal Care and Use Committee (IACUC) at Temple University (Philadelphia, PA) approved all procedures detailed in this section that required the use of vertebrate animals prior to initiating any experimental objectives. Animals were weighed prior to surgery to obtain a baseline for monitoring animal wellness following CCI procedures. Animals were anesthetized using a 1:2 solution of ketamine $(100 \mathrm{mg} / \mathrm{ml})$ to xylazine $(10 \mathrm{mg} / \mathrm{ml}$; Henry Schein Animal Health; Dublin, $\mathrm{OH}$ ) administered by intraperitoneal (IP) injection at a dose of $1 \mathrm{ml} / \mathrm{kg}$. Depth of anesthesia was monitored throughout the surgical procedure by hind paw toe pinches to assure that animals remained properly anesthetized. Animals were shaved to remove hair from the scalp surrounding the surgical area and immobilized using a Mouse ${ }^{\mathrm{TM}}$ Stereotaxic Instrument (Stoelting Co.; Wood Dale, IL). Ophthalmic ointment (Dechra Veterinary Products; Overland Park, KS) was applied to the eyes to prevent ocular drying. Seventy percent isopropyl alcohol was used to clean the scalp, neck, and ears. A Zeiss Stemi 2000C stereomicroscope (Carl Zeiss Microscopy, LLC; Thornwood, NY) equipped with a Schott EasyLED Ringlight (SCHOTT North America Inc.; Elmsford, NY) was used to magnify and illuminate the surgical site. Surgical instruments were autoclaved prior to use and sterilized between animals using a Hot Bead Sterilizer (Fine Science Tools Inc.; Foster City, CA). Surgical scissors were used to remove a portion of the scalp and expose the skull from the sagittal suture to the right temporalis muscle. The underlying fascia was removed, and an Ideal Micro-Drill ${ }^{\mathrm{TM}}$ (CellPoint Scientific Inc.; Gaithersburg, MD) with a $0.5 \mathrm{~mm}$, rounded burr was used to create a $4 \mathrm{~mm}$ craniotomy between bregma and lambda suture lines. The surgical area was periodically washed with $1 \mathrm{X}$ PBS, and drill time was minimized in order to avoid overheating of surrounding tissue. The bone fragment resulting from the craniotomy was carefully lifted away from the underlying brain to avoid disrupting the dura and cortical tissue. Any animal sustaining additional injury to the brain during craniotomy procedures was excluded from the study. An Impact One $^{\mathrm{TM}}$ Stereotaxic CCI Instrument (Leica Microsystems; Buffalo Grove, IL) outfitted with a piston ( $2 \mathrm{~mm}$ diameter) was secured to the stereotaxic stage and positioned over the exposed parietal somatosensory cortex. The piston was oriented parallel to the cortical plane and lowered until contact was made with the dural surface. TBI was delivered with the following parameters: impact velocity: $5 \mathrm{~m} / \mathrm{s}$, impact depth: $1 \mathrm{~mm}$, impactor tip diameter: $2 \mathrm{~mm}$, dwell time: $0.50 \mathrm{~s}$. After discharging the impactor piston, the site of injury was covered by a sterile, $5 \mathrm{~mm}$ glass coverslip (Electron Microscopy Sciences; Hatfield, PA), which was secured to the skull using Vetbond ${ }^{\mathrm{TM}}$ tissue adhesive $(3 \mathrm{M}$; St. Paul, $\mathrm{MN}$ ), creating a waterproof seal between the glass coverslip and the surgical margins of the scalp. After adhering the coverslip atop the craniotomy, animals were removed from the stereotaxic stage and place in their home cage, resting upon an isothermal pad (Braintree Scientific, Inc.; Braintree, MA) to maintain body temperature during recovery. Animals were monitored in their home cage until consciousness was regained. Surgical control (craniotomy only) procedures included all of the steps described above except impactor discharge. Blood was collected from the inferior vena cava into an insulin syringe containing $20 \mu \mathrm{l}$ of $0.5 \mathrm{M}$ EDTA to prevent clotting. Whole blood was spun at $1500 \times \mathrm{g}$ for $20 \mathrm{~min}$ and plasma was transferred to a sterile microcentrifuge tube, snap frozen, and stored at $-80^{\circ} \mathrm{C}$ for later use.

\section{Flow Cytometry of eMVs from Blood Plasma}

eMVs were isolated using the Exoquick system (System Biosystems, Inc.) following the manufacturer's protocol. In short, blood plasma was incubated with Exoquick for $30 \mathrm{~min}$ at $4^{\circ} \mathrm{C}$. After isolation, eMVs were centrifuged at $1500 \times \mathrm{g}$ for $30 \mathrm{~min}$ and resuspended in $100 \mu \mathrm{L}$ of flow cytometry buffer with primary antibody Occludin (abcam) for $1 \mathrm{~h}$. FITC conjugated Secondary antibody (eBioscience) was added for $30 \mathrm{~min}$. $200 \mu \mathrm{L}$ of flow cytometry buffer was added and the samples were analyzed by flow cytometry. Events were acquired for each sample with a FACS BD Canto II flow cytometer (BD Biosciences) and analyzed with FlowJo software (Tree Star, Ashland, OR, USA).

\section{Cryo-EM of eMVs from Blood Plasma Post-TBI}

Isolated eMVs were resuspended in $100 \mu \mathrm{L}$ of buffer containing $20 \mathrm{mM}$ Tris pH 7.4 and $150 \mathrm{mM} \mathrm{Na} / \mathrm{KCl}$. eMVs were incubated with rabbit anti-occludin antibody (abcam) for $1 \mathrm{~h}$ followed by incubation of anti-rabbit IgG (H\&L) conjugated to $10 \mathrm{~nm}$ gold nanoparticles (Cytodiagnostics Inc., Ontario, Canada) for $30 \mathrm{~min}$. eMVs were prepared by high pressure freezing (or cryo-frozen) and analyzed by electron microscopy (EM) at the Electron Microscopy Resource Laboratory (EMRL) headed by Dr. Dewight Williams with technical support by Ray Meade. The EMRL is part of the University of Pennsylvania Biomedical Research Core Facilities.

\section{Statistical Analysis}

The experiments were independently performed multiple times (at least three times for all the data shown) to allow statistical analyses. Within each individual experimental set, every condition was evaluated in three replicates. The data collected were analyzed using Prism v6.0c (GraphPad Software, San Diego, CA). Multiple group comparisons were performed by two-way analysis of variance (ANOVA) with post-hoc analysis or students $t$-test where indicated. All the results are expressed as the mean \pm SEM with differences considered significant at $p<0.05$. 


\section{RESULTS}

\section{Characterization of Brain Endothelial Status Following an in vitro Model of Mechanical Injury}

To model the type of linear kinetic forces that lead to stretchingstrain deformation of tissue during the primary phase of TBI, a Flexcell ${ }^{\circledR}$ Tension System FX-5000 (from Flexcell International Corp.) was utilized. This approach tested the ability of mature monolayers of primary human brain microvascular endothelial cells (BMVECs) to sustain rapid changes in mechanical strain for which micro-tears would be the observable outcome. In addition, this model utilizes a single layer of BMVECs and mimics capillary walls, which are likely key sites of vulnerability in mechanical injury during TBI. Similarly to physiological conditions, mature BMVECs (as described previously) in the above culture conditions display all normal BBB characteristics. Therefore, BMVECs grown on collagen type I coated silicone membranes were subjected to biaxial strain generated by vacuum pump negative pressure, (Figure 1A schematic of cells at rest, top, and under strain, bottom). Figure 1B shows the degree of deformation (membrane elongation profile) when mechanical stimulation was initiated by a semi-sinusoidal tensile stretch pulse lasting $1 \mathrm{~s}$. Profiles are shown for stimulation frequencies of 1.0 and $0.5 \mathrm{~Hz}$, corresponding to membrane elongation of 12 and 22\% respectively. Next, the BMVECs were assayed for the presence of micro-tears after mechanical insult. For better visualization of BMVECs, the cells were loaded with the cell tracker Calcein-AM, which labels and allows the cells to be imaged by fluorescence microscopy. A continuous BMVEC monolayer can be seen in the undisturbed or uninjured control (Figure 3A). In contrast, both the 12 and $22 \%$ elongation increased micro-tear formation between adjacent cells (white arrows). In addition, an increased number of swollen or enlarged cells can readily be seen following the $22 \%$ elongation mechanical injury (yellow arrows). Taken together, these results confirm that strain deformation at either 12 or $22 \%$ elongation induces marked effects to brain endothelial cells in the form of microtears and cellular swelling.

\section{Mechanical Injury Affects Intercellular Tight Junctions (TJs)}

The physical manifestation of the $\mathrm{BBB}$ is due to the presence of tight junction proteins (TJPs) between neighboring endothelial cells (Persidsky et al., 2006). Tearing or dysfunction of the BBB in essence is suggestive of disconnected and disassembled TJs (Aghajanian et al., 2008). To assess whether discontinuity or absence of junctions were evident post mechanical strain, immunocytochemistry analysis of ZO-1 was performed at $2 \mathrm{~h}$ following insult. These studies were also compared with morphological changes, mainly the appearance of stress fibers. As expected in the uninjured control, intense immunopositive staining of the tight junction protein, $\mathrm{ZO}-1$, can be found at the perimeter or junctional areas of every cell (Figure 2). Although ZO-1 is a cytosolic protein, its critical role is to anchor membrane tetraspanin tight junction proteins and thus it
A

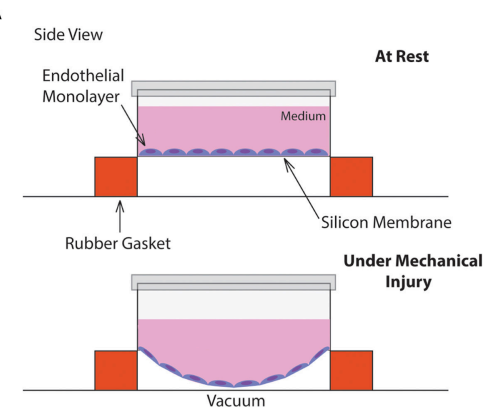

C

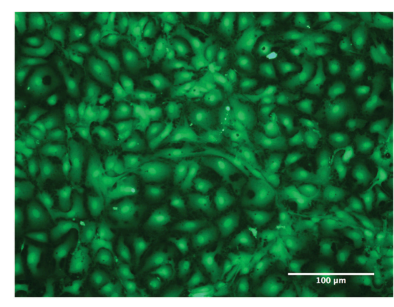

Uninjured

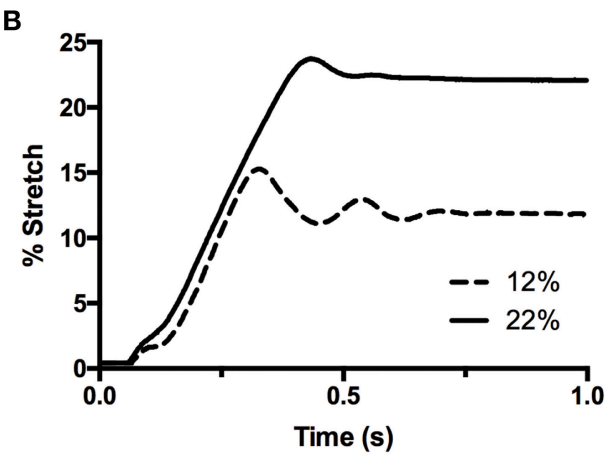

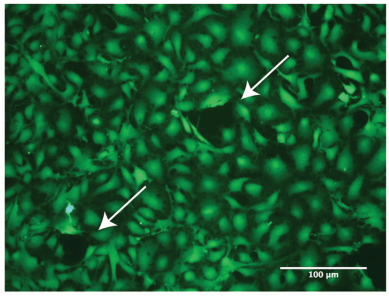

$12 \%$ Stretch

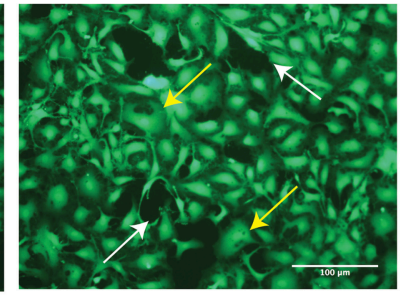

$22 \%$ Stretch

FIGURE 1 | An in vitro model of inducing mechanical biaxial strain. (A) Schematic of the Flexcell ${ }^{\mathrm{TM}}$ injury device. Endothelial cells are grown on elastic silicone membranes that are deformed using negative pressure. (B) Stretch profile of the injury model. Two stretch parameters were used: 12 and $22 \%$ membrane elongation. Injury was performed by applying vacuum over $1 \mathrm{~s}$. (C) Calcein-AM labeling of endothelial cells before and after injury. Uninjured cells are confluent and continuous with neighboring endothelial cells (left). Following 12\% (middle) and 22\% (right) stretch, miro-tears between adjacent endothelial cells develop (white arrows) and at the higher elongation, some cells appear to be swollen (yellow arrows). 


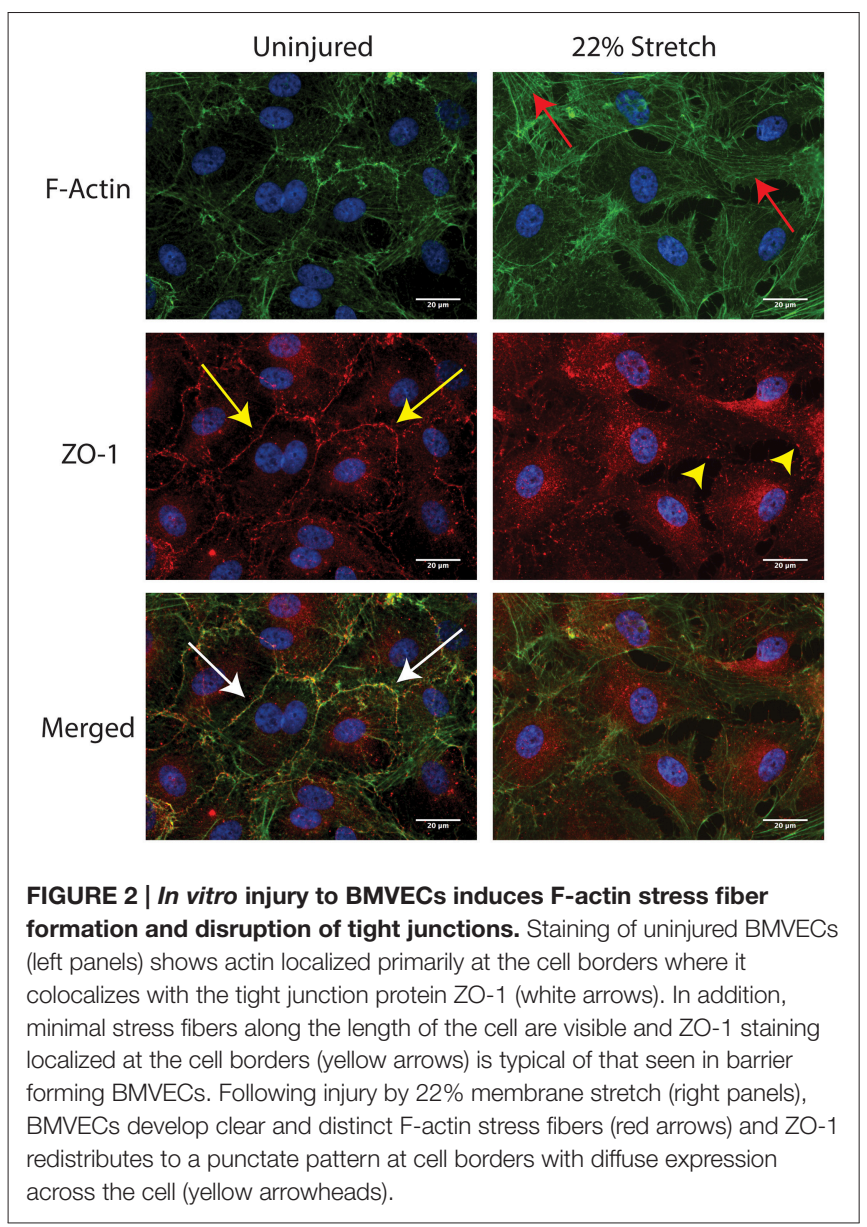

appears junctional (Fanning et al., 1998). Notably, uninjured cells have minimal F-actin stress fiber formation, and instead retain more cortical appearing actin. Meanwhile, BMVECs that had undergone strain-elongation had increased F-actin stress fiber formation (red arrows) and ZO-1 was no longer junctional or co-localized with actin. In addition, the normal intense ZO-1 immunostained pattern became punctate and absent following mechanical insult (yellow arrowhead). Therefore, evaluation of docking TJP, ZO-1 showed that TJ complexes are disrupted as a consequence of increased strain.

To determine whether micro-tears and TJ damage resulting from strain induces changes to metabolic activity, BMVECs were evaluated at the acute $(24 \mathrm{~h})$ phase of mechanical injury with fluorescence reporter PE- $\mathrm{C}_{12}$-resazurin. Analysis of cells by flow cytometry showed that strain decreased metabolic activity as measured by a decrease in the mean fluorescence intensity (MFI) of $\mathrm{PE}-\mathrm{C}_{12}$-resazurin fluorescence (Figures $\mathbf{3 A}, \mathbf{B}$ ). The results showed a 30 and $50 \%$ decrease in mean fluorescence intensity (MFI) for 22 and 12\% stretch, respectively when compared to control. Together these results support the notion that in addition to disrupted tight junctions, changes in cellular energetics also occur due to mechanical injury in BMVECs. Of note, analysis of cell death did not show any significant loss of BMVECs following biomechanical injury at any of the points investigated (Figures 3C,D).

\section{Mechanical Injury Induces a Time-Dependent Release of eMVs Containing TJPs and Endothelial Markers from Brain ECs}

To evaluate whether mechanical injury induces the release of eMVs from BMVECs, BMVECs were subjected to the strain parameters characterized earlier (12 and $22 \%$ stretch) and cells and media were collected at the times indicated. As an initial aspect of the secondary phase of TBI, endothelial cells upregulate surface expression of ICAM-1. This is thought to occur in order to recruit immune cells to sites of inflammation (Whalen et al., 1999). Interestingly, the overall surface expression of ICAM1 , as determined by flow cytometry, decreased on BMVECs following strain, trending downward as early as $2 \mathrm{~h}$ and reaching significance at $6 \mathrm{~h}$. ICAM-1 expression remained decreased at $48 \mathrm{~h}$ post strain (Figures 4A-D). This effect on ICAM-1 surface expression followed the same trend for both mechanical insults tested. Conversely, ICAM-1 expression on shed eMVs isolated from the media was significantly increased, thus inversely correlating to the observation in cellular expression (Figure 4E). Further analysis of the isolated eMVs showed the presence of endothelial marker PECAM-1 and tight junction protein occludin following injury (Figures 5A,B). PECAM-1 expression in eMVs was increased rapidly (at $2 \mathrm{~h}$ ) and persisted at elevated levels (Figure 5A). The increase in occludin, however, was timedependent and peaked at $24 \mathrm{~h}$ post injury (Figure 5B).

The involvement of the small GTP-protein ARF6 in both vascular permeability and microvesicle formation has been recently documented (Muralidharan-Chari et al., 2009; Akers et al., 2013; Ghossoub et al., 2014). Therefore, it was examined whether ARF6 would also play a role in BMVEC eMV shedding following changes in mechanical strain. ARF6 activity was measured using a G-LISA assay that captures ARF6 and then detects ARF6 bound to GTP with another specific antibody. Activation of ARF6 increased significantly by $38 \% 5 \mathrm{~min}$ after the cells were subjected to biaxial strain. Constituently active ARF6 was used as positive control for the assay. (Two-tailed $t$-test average \pm SEM Static $0.3362 \pm 0.0540, n=6,22 \%$ stretch $0.4644 \pm 0.07, n=3{ }^{*} p<0.05$, positive control $0.7279 \pm$ $0.0787, n=2$ ). The results from this assay point to the possible involvement of ARF6 in eMV biogenesis resulting from kinetic forces acting on brain endothelium.

\section{Extracellular Microvesicles (eMVs) Containing Occludin are Detected in Blood Plasma Following TBI}

A recent study has indicated that elevated levels of occludin are detectible in the blood of patients following a mild TBI (Shan et al., 2016). Importantly, the above study did not measure occludin in isolated eMVs. To determine if occludin was released in eMVs following TBI, blood plasma was collected $24 \mathrm{~h}$ after experimental TBI using the controlled-cortical impact (CCI) mouse model. Purified eMVs were analyzed by flow cytometry and cryo-EM. Figures 6A-E, shows the results from flowcytometry analysis comparing the levels of occludin positive eMVs detected in blood plasma of sham versus CCI-TBI mice. 
A

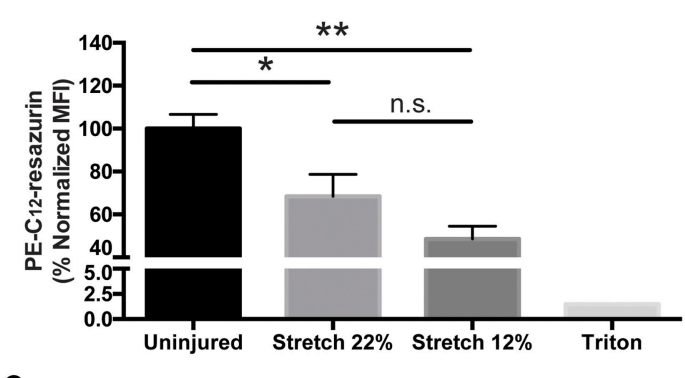

C

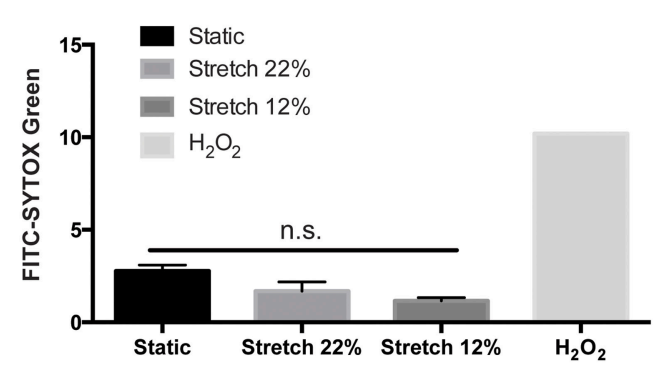

B

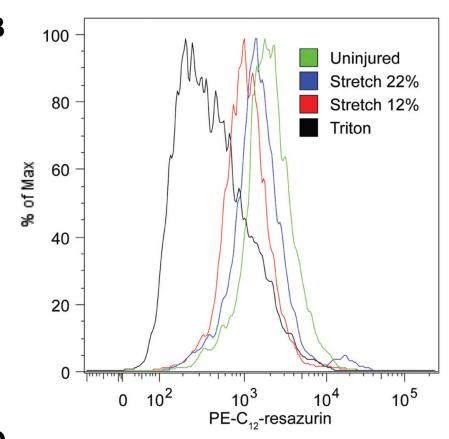

D
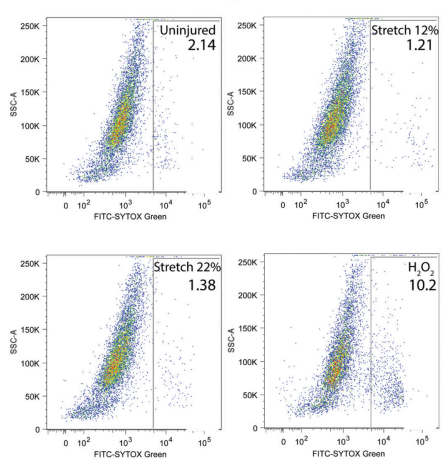

FIGURE 3 | In vitro endothelial injury decreases metabolic activity without inducing cell death in BMVECs. (A) PE mean fluorescence intensity (MFI), a measure of cell metabolic activity, was analyzed in injured and uninjured BMVECs using flow cytometry. Injured cells exhibited reduced red fluorescence intensity reflecting a decrease in metabolic activity, which converts $\mathrm{C}_{12}$-resazurin to red-fluorescent $\mathrm{C}_{12}$-resorufin. $\mathrm{A}$ positive control (0.1\% Triton $\mathrm{X}$ - 100$)$, which disrupts cell membranes and reduces metabolic activity, was used to verify the assay (One-way ANOVA and Tukey post-hoc test average \pm SEM uninjured $n=8,12 \% n=5,22 \%$ $n=6,{ }^{*} p<0.05,{ }^{* *} p<0.01$ ). (B) Representative histograms of PE-C 12 -resazurin MFI for each condition. (C) Cell death was determined using STYOX ${ }^{\circledR}$ Green, a green fluorescent nuclear and chromosome counterstain, was used to determined the percentage of dead cells. A positive control ( 2 mM $\mathrm{H}_{2} \mathrm{O}_{2}$ ) was used to induce cell death. (One-way ANOVA and Sidak post-hoc test average \pm SEM uninjured $n=4,12 \% n=5,22 \% n=6$ ). (D) Representative dot plots are shown for each condition.

Two clear occludin positive populations were detected and were designated as either high or low forward side scatter (FSC). FSC is related to the amount of laser passing around the vesicle and is thus related to its size. Analysis of occludin positive vesicles showed a 5-fold increase high-FSC vesicles (Figure 6C) and a 7.5-fold increase in total occludin positive vesicles (Figure 6B) following CCI-TBI as compared to sham. Size beads (ranging from 0.22 to $1.34 \mu \mathrm{m}$ diameter) were used to estimate the size of occludin positive vesicles (Figure 6D). Occludin positive vesicles appeared to be predominately smaller than $0.45 \mu \mathrm{m}$ diameter. To further characterize the morphology of eMVs produced following TBI, indirect imaging by high-resolution cryo-EM which has previously been used to visualize extracellular vesicles can provide information regarding eMV size, structure, and composition (Issman et al., 2013). As can be seen in the sample cryo-EM image shown (Figure 6E), eMVs had diameters ranging between 50 and $300 \mathrm{~nm}$. Furthermore, immunogold labeling clearly outlined occludin positive vesicles primarily in the size range of 100-300 $\mathrm{nm}$ in diameter (Figure 6E). Of note, not all eMVs in the sample image show immunoreactivity for occludin, indicating the possibility that these eMVs may be from other cellular sources other than brain endothelium. These analyses provide strong in vivo evidence for the presence and robust production (at $24 \mathrm{~h}$ ) of occludin containing eMVs in the blood serum of animals following CCI-TBI. The data also demonstrates for the first time visual confirmation of isolated eMVs highly enriched with the tight junction protein occludin (i.e., as seen by the presence of immunogold antibodies to occludin).

\section{DISCUSSION}

Prior studies have demonstrated the significance of BBB damage following TBI. However, our understanding of the effects of mechanical injury on cerebral vascular endothelium remodeling remains incomplete. We have therefore utilized both in vitro and in vivo approaches to study responses of the cerebral endothelium to mechanical injury. Here we present compelling results that demonstrate that brain endothelial cells release eMVs containing the TJP occludin in response to biaxial strain (as a means to simulate the primary or kinetic induced injured that is seen in TBI) and the controlled cortical impact animal model of TBI.

Devices that involve deformation of a cellular support membrane (Winston et al., 1989; Schaffer et al., 1994; Brown, 2000) are commonly used to study the effects of physiological strain on endothelial cells (Vion et al., 2013a; Figueroa et al., 2014) and TBI-like injury on neurons and glial cells (Ellis et al., 1995; Geddes-Klein et al., 2006; Cullen et al., 2007). Only recently have these devices been used to model the effects of TBI on 


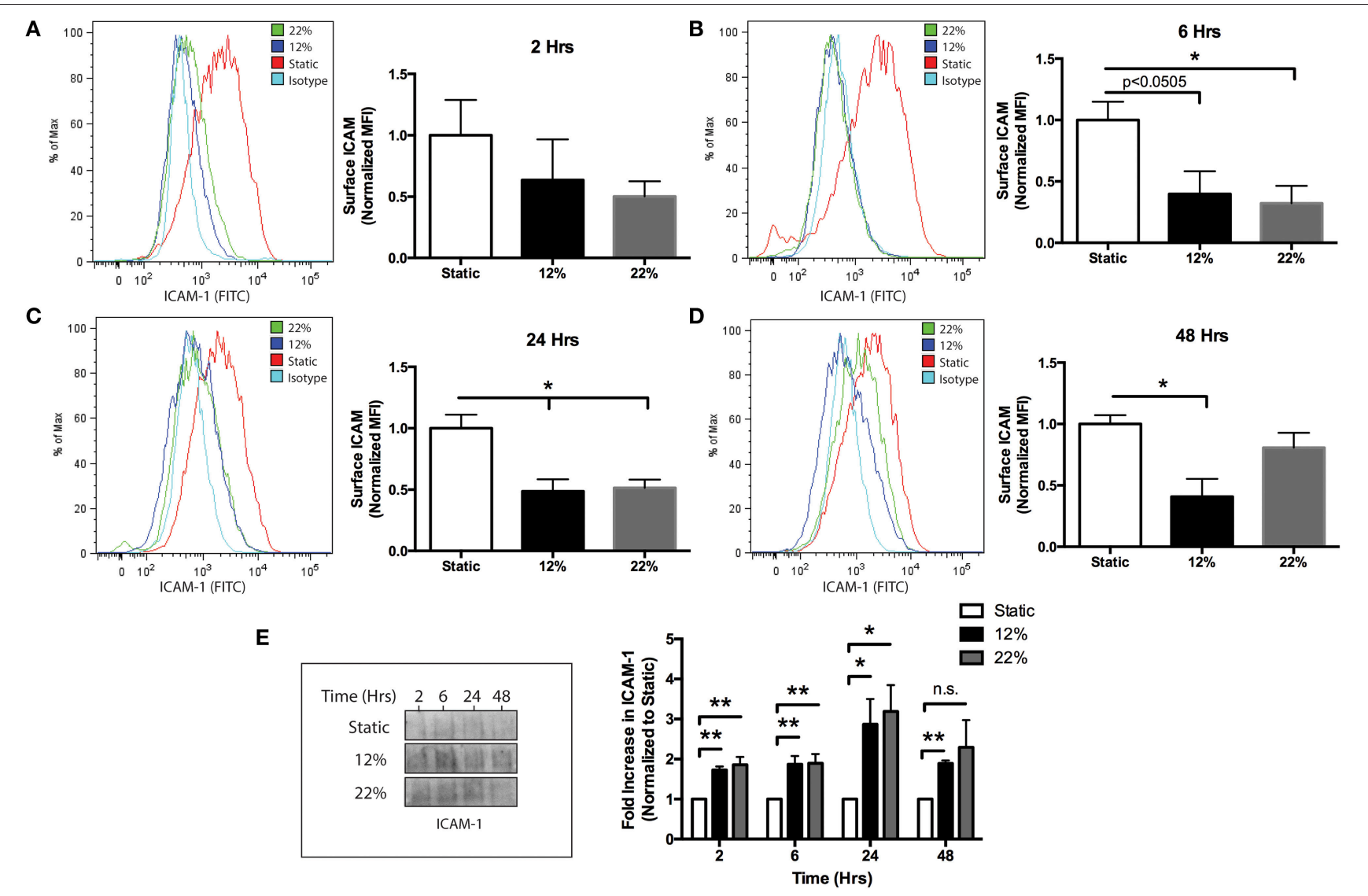

FIGURE 4 | Reduced surface expression of ICAM-1 and induced production of eMVs containing ICAM-1 following in vitro biomechanical injury of brain endothelial cells. (A-D) show surface ICAM-1 expression by flow cytometry, histograms and bar graph representation for the following time points post injury: (A) $2 \mathrm{~h},(\mathbf{B}), 6 \mathrm{~h}$ (C), $24 \mathrm{~h}$ (D) $48 \mathrm{~h}$ (two-tailed $t$-test ${ }^{*} p<0.05$ average $\pm \mathrm{SEM}$ at rest $n=4,12$, and $22 \%$ stretch $n=3$ at $t=2$ and $6 \mathrm{~h}, n=4 t=24 \mathrm{~h}, n=6 t=48 \mathrm{~h}$ ) (E) eMVs were collected from the media at the indicated time points and analyzed by western blot. Injured cells released greater quantities of ICAM-1, which was time and stretch dependent. (Two-way ANOVA and students $t$-test ${ }^{*} p<0.05$, ${ }^{* *} p<0.01$, average \pm SEM Static $n=5,12 \% n=4$, and $22 \% n=5$ except one outlier was excluded for $12 \% t=48 \mathrm{~h}$ and $22 \% t=2 \mathrm{~h}$ using Grubb's test $p<0.05$ ).

endothelial cells (Salvador et al., 2013, 2015). Characterization of the injury induced from the in vitro mechanical strain model used in this study reproduces the type of linear kinetic forces that induces micro-tears to the endothelium in TBI (Figure 1). Indeed we also observed that mechanical injury induced changes in metabolism resulting from membrane damage (Figures 3A,B). While not significantly significant, there was a trend for a greater decrease in metabolic activity from $12 \%$ substrate elongation as compared to $22 \%$ elongation. It is unclear if this is a result of small differences in the membrane elongation profile achieved using our experimental set-up or if it is a real endothelial physiological response to mechanical injury. Furthermore, stretch injury models such as these typically do not increase cell death even on neuronal cells subjected to strain/stretch ranging from 0 to $50 \%$ (Geddes-Klein et al., 2006). We also saw no increase in cell death following substrate elongation (Figures 3C,D). Instead, many endothelial cells appeared to be swollen following injury (Figure 1), which has been reported by others both in vitro (Salvador et al., 2013, 2015) and in vivo (Simard et al., 2010). This change in cell volume is theorized to alter cytoskeletal dynamics and weaken tight junctions leading to the increased barrier permeability (Simard et al., 2010).

Short-term studies $(24-72 \mathrm{~h})$ have found that in vitro and in vivo models of TBI decrease endothelial expression of TJPs (occludin, ZO-1, and Cld-5; Higashida et al., 2011; Hue et al., 2013; Wen et al., 2014; Salvador et al., 2015) and result in discontinuous ZO-1 staining in histological analysis of brain tissue (Thal et al., 2012). We also observed disrupted tight junctions and a transition of ZO-1 expression from continuous localization along cell borders to a discontinuous, punctate, and even absent expression pattern (Figure 2). These changes in ZO-1 localization and subcellular distribution were accompanied by decreased colocalization with F-actin (Figure 2). The colocalization of F-actin with TJPs has been well documented, and actin has been proposed to play a critical role in maintaining TJ complex integrity and stability (Lai et al., 2005). While TJP disassembly and expression changes have been reported to occur following TBI, it has been unclear whether TJPs are degraded internally or processed and packaged to be released extracellularly. Here we present for the first time evidence that 

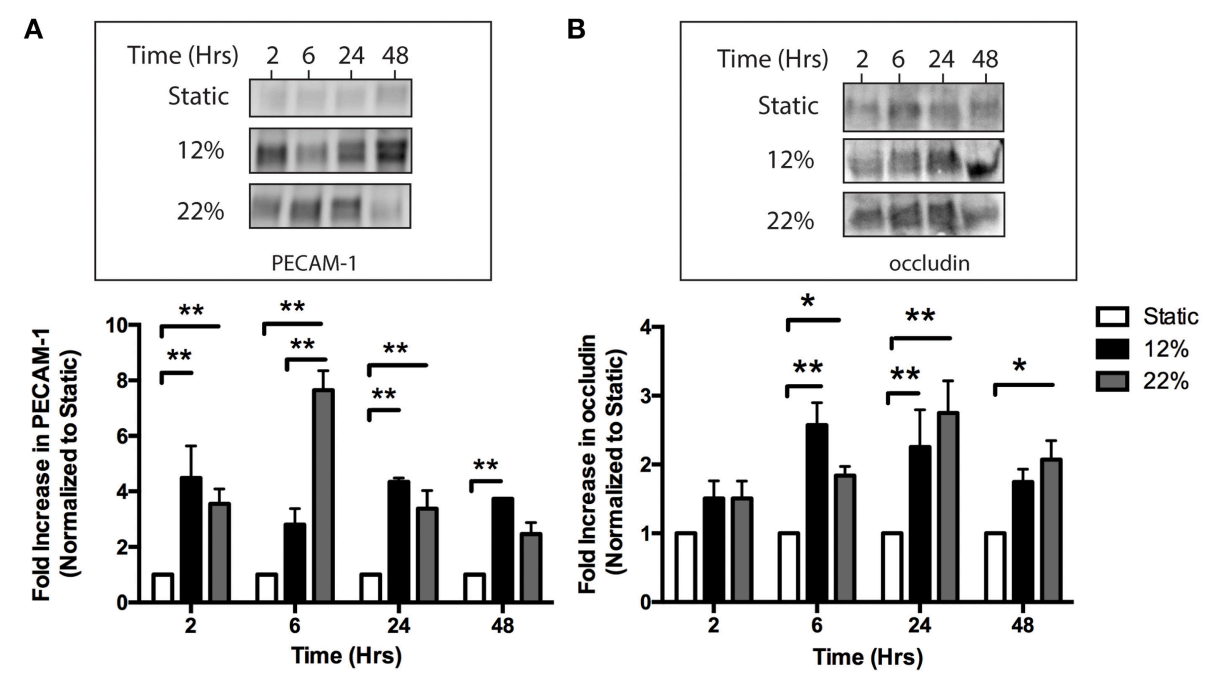

FIGURE 5 | In vitro mechanical injury induces the release of eMVs containing the endothelial marker PECAM-1 and tight junction protein occludin. (A) Injury increases the release of eMVs containing PECAM-1. eMVs were isolated from media using Exoquick and analyzed by western blot. Elevated levels of PECAM- 1 were detected at $2 \mathrm{~h}$ and remained elevated for most time points. (Two-way ANOVA with Bonferroni post-hoc analysis * $p<0.01$ average \pm SEM Static and $22 \% n=6,12 \% n=4)(B)$ Injury induces a time-dependent increase in eMVs containing occludin. eMVs were isolated from the media using Exoquick at the indicated time points post-injury and analyzed by western blot. Statistically significant increases were seen at 6 , 24 , and $48 \mathrm{~h}$ after injury. (Two-way ANOVA with Bonferroni post-hoc analysis ${ }^{*} p<0.05,{ }^{* *} p<0.01$ average \pm SEM Static and $22 \% n=6,12 \% n=4$ except for $12 \%$ $t=24 \mathrm{~h}$ where one outlier was excluded using Grubb's test $p<0.05)$.

both in vitro, via mechanically induced strain (Figure 5), and in vivo, using a leading model of experimental TBI (Figure 6), mechanical injury increases the release of eMVs containing the TJP occludin.

The endothelium is constantly exposed to mechanical forces such as shear stress and strain and consequently remodels in response to these mechanical forces. The production of eMVs by endothelial cells following mechanical stimuli has only recently been investigated in the context of physiological shear stress and cyclic strain in vitro (Vion et al., 2013a,b; Letsiou et al., 2015) and in vivo (Jenkins et al., 2013). Other studies have shown that human pulmonary and umbilical endothelial cells (HUVECs) respond to mechanical forces to produce microparticles and that the mechanism of cyclic stretch-induced vesicle release is thought to be activated by the release of TNF- $\alpha$ and other cytokines (Wang et al., 2003; Dignat-George and Boulanger, 2011). A recent study using an in vitro TBI model on endothelial cells showed an increase in TNF- $\alpha$ and CCL 2 mRNA production $24 \mathrm{~h}$ post injury (Salvador et al., 2015). TNF- $\alpha$ and other inflammatory cytokines are known to induce the upregulation of ICAM-1 in brain ECs (Ramirez et al., 2012; Rom et al., 2015). Therefore, we anticipated that ICAM-1 surface expression would be upregulated following mechanical injury. However, we observed a decrease in ICAM-1 surface staining, which corresponded to an increase in shedding of ICAM-1 in eMVs (Figure 4). Upregulation of ICAM-1 and other adhesion molecules by the cerebral endothelium following a TBI is well established (Carlos et al., 1997). An important distinction between our work and previous studies utilizing in vitro TBI models is that our data reflects changes in primary human BMVECs vs. an immortalized murine brain ECs line (Salvador et al., 2013, 2015). In addition, Salvador et al. (2015) detected increased mRNA for inflammatory cytokines but they did not evaluate other indices of endothelial activation such as cytokine release or protein expression of adhesion molecules (Salvador et al., 2015). The lack of ICAM-1 induction in brain ECs has led us to hypothesize that in the absence of other neurovascular unit (NVU) members (i.e., microglia, astrocytes), brain ECs likely initiate repair or angiogenic mechanisms without activation. This is supported by studies showing the production of by cytokines produced by other cells of the NVU (Carlos et al., 1997), a contribution which is lacking in our in vitro experimental model. Therefore, as basal cellular surface levels of ICAM-1 decrease via shedding of eMVs, brain ECs may only upregulate ICAM-1 in response to signals from other injured cells (i.e., microglia, astrocytes, and neurons) or by recruited immune cells. In summary, increased eMV production in response to mechanical injury was observed in both in vitro and in vivo models and represents an understudied aspect of the consequences of TBI.

The profile of ICAM-1 release in eMVs was similar at both 12 and $22 \%$ elongation while the profile of PECAM-1 and occludin had differential changes. Specifically, at $12 \%$ elongation, PECAM-1 release remained relatively constant at all time points while $22 \%$ elongation induced a graded release that peaked at $6 \mathrm{~h}$. Occludin expression in eMVs was also a graded response, which peaked earlier in response to $12 \%$ as compared to $22 \%$ elongation. While it might be expected that eMV release would be linear in response to mechanical injury, where greater injury induces greater release for all proteins, little is known regarding this process. Our results provides some initial insight into the 


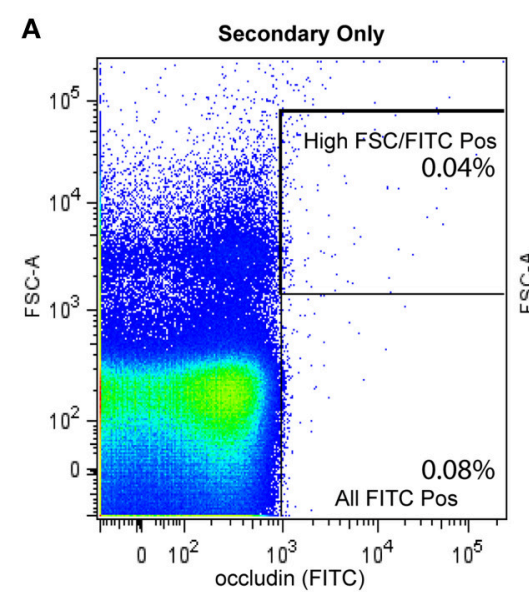

B

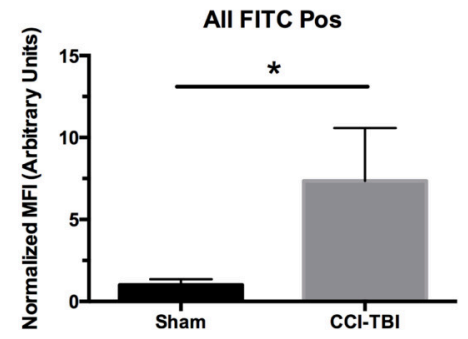

C

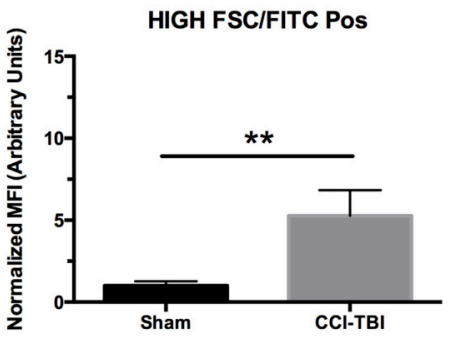

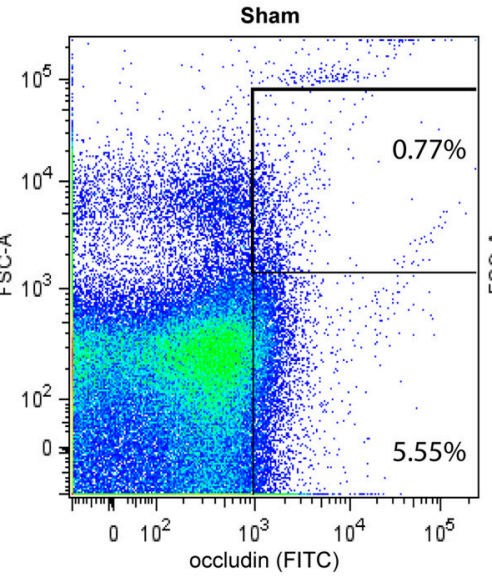

D

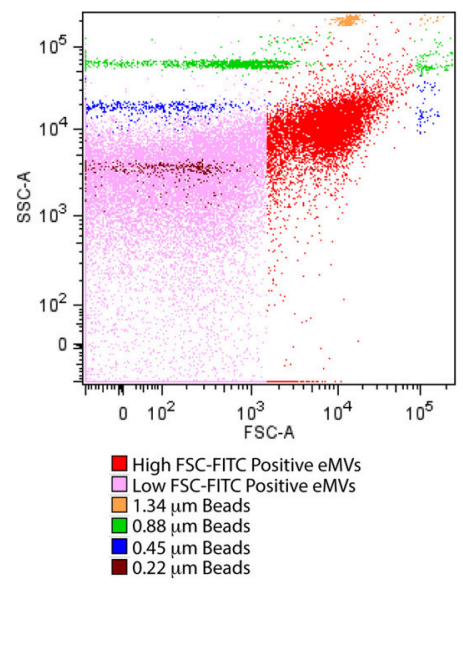

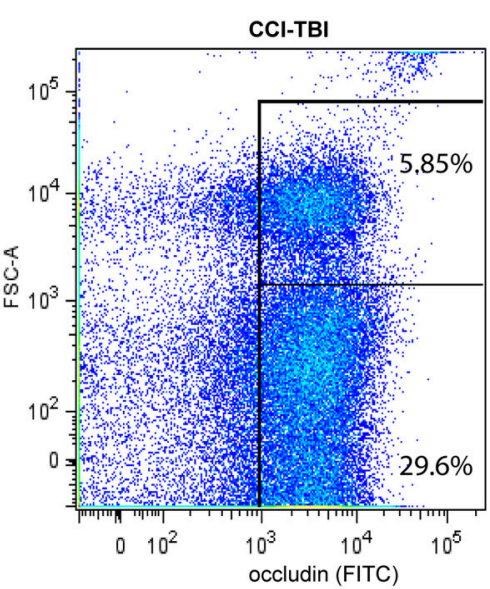

E

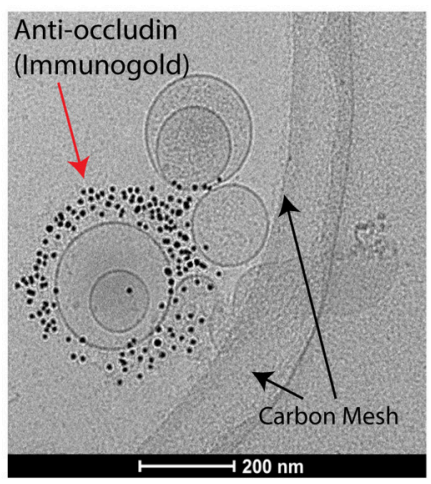

FIGURE 6 | Experimental TBI induces the release of eMVs containing the tight junction protein occludin. Mice were subjected to sham surgery or a Controlled Cortical Impact, CCI-TBI and blood was collected $24 \mathrm{~h}$ post-injury. (A) Flow Cytometry detection of occludin (FITC) positive eMVs from blood plasma. Regions of interest were drawn around all FITC positive vesicles as well as a clear high FSC population. (B,C) TBI induces an increase in total FITC positive and high FSC-FITC positive vesicles at $24 \mathrm{~h}$ post-injury (Students two-tailed $t$-test average \pm SEM $n=5$ for sham and $n=4$ for TBI, ${ }^{*} p<0.05$, ${ }^{* *} p<0.01$, one was excluded from the sham group for being a significant outlier). (D) Occudin (FITC) positive vesicles (shown in red) were compared to beads of known size ranging from 0.22 to $1.34 \mu \mathrm{m}$ in diameter. (E) eMVs isolated post-TBI were analyzed using cryo-EM and immunogold labeling to show occludin positive microvesicles.

response of brain ECs to strain in vitro and in vivo, and further investigation would provide a more thorough understand of the kinetics of eMV release from mechanical injury.

Regarding extracellular vesicles, two main categories have been identified based on their size and mechanism of release. Microparticles (MPs) are the larger of the two categories ranging between 0.1 and $1 \mu \mathrm{m}$ in diameter and are generated by outward blebbing of the plasma membrane. Exosomes are smaller (30$100 \mathrm{~nm}$ ) and are released by vesicular fusion with the plasma membrane (Raposo and Stoorvogel, 2013). In this study we have not focused on identifying the type of extracellular vesicle that contains the TJP occludin or endothelial markers (PECAM-1, ICAM-1). A number of studies have reported on the localization of both PECAM-1 and ICAM-1 in MPs (Jimenez et al., 2003). Our flow cytometry data of occludin positive eMVs from blood plasma (Figure 6A) identified two clear populations based on size. However, standard flow cytometers do not have the resolution to accurately identify exosomes based on their small size. Thus it is unclear if the two populations reported by flow cytometry here translate to the two types of vesicles. Occludinimmunogold labeling and cryo-EM imaging (Figure 6E) showed occludin positive vesicles that fall into the size category of both types of extracellular vesicles. Aside from identifying occludin positive vesicles, a number of vesicles detected by these methods appeared to be multivesicular which has been shown previously (Issman et al., 2013). Of note, the detection of immunogold labeled vesicles that appeared to be inside another vesicle likely reflects two vesicles stacked on top of one another (arrow) instead of a multivesicular vesicle (arrowhead). In addition, some vesicles appeared to be more granular than others which suggests differences in content (Issman et al., 2013). 
The mechanism of extracellular vesicle release has been linked to cytoskeletal rearrangement (Boulanger et al., 2006; Latham et al., 2013) as well as small GTPases such as Rho (Burger et al., 2011) and ARF6 (Pasquier et al., 2014). ARF6 has been linked to both microvesicles (Pasquier et al., 2014) and exosome release (Friand et al., 2015). In the case of microvesicles, ARF6 activation leads to downstream phospholipase D and ERK signaling, MLC activation and finally acto-myosin contraction at the vesicle neck and the release into the extracellular space (MuralidharanChari et al., 2009). For exosomes, ARF6 and phospholipase D2 control the budding of intraluminal vesicles into multivesicular bodies. Multivesicular bodies will later fuse with the membrane to release the exosomes extracellularly (Ghossoub et al., 2014). Based on the evidence for ARF6 involvement in extracellular vesicle release we analyzed ARF6 activation and found that it was elevated following injury (Results Section). The activation of ARF6 and detection of eMVs following injury contributes to our understanding of the effects of mechanical damage to the endothelium.

We believe that the results presented here represent a significant advancement to our understanding of cerebral vascular remodeling following TBI. In addition, our approach to understanding the effects of TBI is highly BBB and endothelial centric and offers the potential to uncover new biomarkers for the monitoring and diagnosis of TBI. The field of TBI research has indeed recognized the lack of available effective biomarkers for diagnosing and monitoring a patient following brain injury (Forde et al., 2014), and many prior studies have focused on biomarkers related to neuronal and astrocytic damage with few focused on biomarkers specific for BBB damage. Cardiovascular research has spearheaded studies focusing specifically on endothelial damage and the production of extracellular vesicles as a diagnostic and prognostic tool for hypertension, atherosclerosis, diabetes, and endothelial dysfunction (Boulanger et al., 2006; Chironi et al., 2009).

\section{REFERENCES}

Abbott, N. J., Ronnback, L., and Hansson, E. (2006). Astrocyte-endothelial interactions at the blood-brain barrier. Nat. Rev. Neurosci. 7, 41-53. doi: $10.1038 / \mathrm{nrn} 1824$

Aghajanian, A., Wittchen, E. S., Allingham, M. J., Garrett, T. A., and Burridge, K. (2008). Endothelial cell junctions and the regulation of vascular permeability and leukocyte transmigration. J. Thromb. Haemost. 6, 1453-1460. doi: 10.1111/j.1538-7836.2008.03087.x

Akers, J. C., Gonda, D., Kim, R., Carter, B. S., and Chen, C. C. (2013). Biogenesis of extracellular vesicles (EV): exosomes, microvesicles, retrovirus-like vesicles, and apoptotic bodies. J. Neurooncol. 113, 1-11. doi: 10.1007/s11060-013-1084-8

Bernas, M. J., Cardoso, F. L., Daley, S. K., Weinand, M. E., Campos, A. R., Ferreira, A. J., et al. (2010). Establishment of primary cultures of human brain microvascular endothelial cells to provide an in vitro cellular model of the blood-brain barrier. Nat. Protoc. 5, 1265-1272. doi: 10.1038/nprot.2010.76

Boshans, R. L., Szanto, S., van Aelst, L., and D'Souza-Schorey, C. (2000). ADP-ribosylation factor 6 regulates actin cytoskeleton remodeling in coordination with Rac1 and RhoA. Mol. Cell. Biol. 20, 3685-3694. doi: 10.1128/MCB.20.10.3685-3694.2000

Boulanger, C. M., Amabile, N., and Tedgui, A. (2006). Circulating microparticles: a potential prognostic marker for atherosclerotic vascular disease. Hypertension 48, 180-186. doi: 10.1161/01.HYP.0000231507.00962.b5
Numerous studies have shown that endothelial cells (pulmonary, umbilical, artery, aortic) release eMVs in response to a number of insults including inflammatory cytokines, mechanical injury, and serum deprivation (Chironi et al., 2009). In these studies, eMV levels are typically determined by Annexin V (microparticles; Draeger et al., 2011) or exosome markers (CD63, Alix, etc.), which are general markers and are therefore not cell or organ specific. Some studies have proposed the differentiation of the cell lineage by cell specific markers such as P-selectin (CD62P) and CD63 for platelets (Tan et al., 2005) and PECAM-1 (CD31) for endothelial cells (Boulanger et al., 2006). Here, we propose an alternative method of eMV detection and identification by TJPs. Coupled with endothelial markers and other proteins enriched at the $\mathrm{BBB}$, this approach delineates $\mathrm{MV}$ origin, primarily from brain ECs, and also has the potential to reflect the functionality of the host cell and provide a clinically useful assessment of BBB status in TBI. Detection of brain endothelial-derived eMVs could provide a novel means to analytically evaluate $\mathrm{BBB}$ health in a number of CNS conditions that are associated with trauma and neuroinflammation.

\section{AUTHOR CONTRIBUTIONS}

AA and SR contributed to the study design. AA, EL, SM conducted the experiments. AA, SR, EL, SM, RR contributed to the writing of the manuscript.

\section{ACKNOWLEDGMENTS}

We would like to thank the Penn Core Facility (particularly Ray Meade and Dr. Dewight Williams) for their help with cryo-EM. This study was supported (in part) by research funding from the NIH: 1R01NS086570 (to SHR) and the Shriners Hospitals for Children: 85110-PHI-14 (to SHR), NIH-NIDA T32 DA007237 (AA), and P30-DA13429 (SHR).
Brown, T. D. (2000). Techniques for mechanical stimulation of cells in vitro: a review. J. Biomech. 33, 3-14. doi: 10.1016/S0021-9290(99) 00177-3

Burger, D., Montezano, A. C., Nishigaki, N., He, Y., Carter, A., and Touyz, R. M. (2011). Endothelial microparticle formation by angiotensin II is mediated via Ang II receptor type I/NADPH oxidase/ Rho kinase pathways targeted to lipid rafts. Arterioscler. Thromb. Vasc. Biol. 31, 1898-1907. doi: 10.1161/ATVBAHA.110.222703

Carlos, T. M., Clark, R. S., Franicola-Higgins, D., Schiding, J. K., and Kochanek, P. M. (1997). Expression of endothelial adhesion molecules and recruitment of neutrophils after traumatic brain injury in rats. J. Leukoc. Biol. 61, 279-285.

Chironi, G. N., Boulanger, C. M., Simon, A., Dignat-George, F., Freyssinet, J. M., and Tedgui, A. (2009). Endothelial microparticles in diseases. Cell Tissue Res. 335, 143-151. doi: 10.1007/s00441-008-0710-9

Cullen, D. K., Stabenfeldt, S. E., Simon, C. M., Tate, C. C., and LaPlaca, M. C. (2007). In vitro neural injury model for optimization of tissue-engineered constructs. J. Neurosci. Res. 85, 3642-3651. doi: 10.1002/jnr.21434

Dignat-George, F., and Boulanger, C. M. (2011). The many faces of endothelial microparticles. Arterioscler. Thromb. Vasc. Biol. 31, 27-33. doi: 10.1161/ATVBAHA.110.218123

Draeger, A., Monastyrskaya, K., and Babiychuk, E. B. (2011). Plasma membrane repair and cellular damage control: the annexin survival kit. Biochem. Pharmacol. 81, 703-712. doi: 10.1016/j.bcp.2010.12.027 
Ellis, E. F., McKinney, J. S., Willoughby, K. A., Liang, S., and Povlishock, J. T. (1995). A new model for rapid stretch-induced injury of cells in culture: characterization of the model using astrocytes. J. Neurotrauma 12, 325-339. doi: 10.1089/neu.1995.12.325

Fanning, A. S., Jameson, B. J., Jesaitis, L. A., and Anderson, J. M. (1998). The tight junction protein ZO-1 establishes a link between the transmembrane protein occludin and the actin cytoskeleton. J. Biol. Chem. 273, 29745-29753. doi: $10.1074 /$ jbc. 273.45.29745

Figueroa, D. S., Kemeny, S. F., and Clyne, A. M. (2014). Glycated collagen decreased endothelial cell fibronectin alignment in response to cyclic stretch via interruption of actin alignment. J. Biomech. Eng. 136, 101010. doi: $10.1115 / 1.4028037$

Forde, C. T., Karri, S. K., Young, A. M., and Ogilvy, C. S. (2014). Predictive markers in traumatic brain injury: opportunities for a serum biosignature. $\mathrm{Br}$. J. Neurosurg. 28, 8-15. doi: 10.3109/02688697.2013.815317

Friand, V., David, G., and Zimmermann, P. (2015). Syntenin and syndecan in the biogenesis of exosomes. Biol. Cell 107, 331-341. doi: 10.1111/boc.201500010

Geddes-Klein, D. M., Schiffman, K. B., and Meaney, D. F. (2006). Mechanisms and consequences of neuronal stretch injury in vitro differ with the model of trauma. J. Neurotrauma 23, 193-204. doi: 10.1089/neu.2006.23.193

Ghossoub, R., Lembo, F., Rubio, A., Gaillard, C. B., Bouchet, J., Vitale, N., et al. (2014). Syntenin-ALIX exosome biogenesis and budding into multivesicular bodies are controlled by ARF6 and PLD2. Nat. Commun. 5, 3477. doi: $10.1038 /$ ncomms4477

Glushakova, O. Y., Johnson, D., and Hayes, R. L. (2014). Delayed increases in microvascular pathology after experimental traumatic brain injury are associated with prolonged inflammation, blood-brain barrier disruption, and progressive white matter damage. J. Neurotrauma 31, 1180-1193. doi: 10.1089/neu.2013.3080

Higashida, T., Kreipke, C. W., Rafols, J. A., Peng, C., Schafer, S., Schafer, P., et al. (2011). The role of hypoxia-inducible factor-1alpha, aquaporin4, and matrix metalloproteinase-9 in blood-brain barrier disruption and brain edema after traumatic brain injury. J. Neurosurg. 114, 92-101. doi: 10.3171/2010.6.JNS10207

Hue, C. D., Cao, S., Haider, S. F., Vo, K. V., Effgen, G. B., Vogel, E. III, et al. (2013). Blood-brain barrier dysfunction after primary blast injury in vitro. J. Neurotrauma 30, 1652-1663. doi: 10.1089/neu.2012.2773

Issman, L., Brenner, B., Talmon, Y., and Aharon, A. (2013). Cryogenic transmission electron microscopy nanostructural study of shed microparticles. PLoS ONE 8:e83680. doi: 10.1371/journal.pone.0083680

Jenkins, N. T., Padilla, J., Boyle, L. J., Credeur, D. P., Laughlin, M. H., and Fadel, P. J. (2013). Disturbed blood flow acutely induces activation and apoptosis of the human vascular endothelium. Hypertension 61, 615-621. doi: 10.1161/HYPERTENSIONAHA.111.00561

Jimenez, J. J., Jy, W., Mauro, L. M., Soderland, C., Horstman, L. L., and Ahn, Y. S. (2003). Endothelial cells release phenotypically and quantitatively distinct microparticles in activation and apoptosis. Thromb. Res. 109, 175-180. doi: 10.1016/S0049-3848(03)00064-1

Korn, A., Golan, H., Melamed, I., Pascual-Marqui, R., and Friedman, A. (2005). Focal cortical dysfunction and blood-brain barrier disruption in patients with Postconcussion syndrome. J. Clin. Neurophysiol. 22, 1-9. doi: 10.1097/01.WNP.0000150973.24324.A7

Lai, C. H., Kuo, K. H., and Leo, J. M. (2005). Critical role of actin in modulating BBB permeability. Brain Res. Brain Res. Rev. 50, 7-13. doi: 10.1016/j.brainresrev.2005.03.007

Latham, S. L., Chaponnier, C., Dugina, V., Couraud, P. O., Grau, G. E., and Combes, V. (2013). Cooperation between beta- and gamma-cytoplasmic actins in the mechanical regulation of endothelial microparticle formation. FASEB J. 27, 672-683. doi: 10.1096/fj.12-216531

Letsiou, E., Sammani, S., Zhang, W., Zhou, T., Quijada, H., Moreno-Vinasco, L., et al. (2015). Pathologic mechanical stress and endotoxin exposure increases lung endothelial microparticle shedding. Am. J. Res. Cell Mol. Biol. 52, 193-204. doi: $10.1165 / \mathrm{rcmb} .2013-0347 \mathrm{OC}$

Liu, W., Soderlund, K., Senseney, J. S., Joy, D., Yeh, P. H., Ollinger, J., et al. (2016). Imaging cerebral microhemorrhages in military service members with chronic traumatic brain injury. Radiology 278, 536-545. doi: 10.1148/radiol.2015150160

Muralidharan-Chari, V., Clancy, J., Plou, C., Romao, M., Chavrier, P., Raposo, G., et al. (2009). ARF6-regulated shedding of tumor cell-derived plasma membrane microvesicles. Curr. Biol. 19, 1875-1885. doi: 10.1016/j.cub.2009. 09.059

Pasquier, J., Thawadi, H. A., Ghiabi, P., Abu-Kaoud, N., Maleki, M., Guerrouahen, B. S., et al. (2014). Microparticles mediated cross-talk between tumoral and endothelial cells promote the constitution of a pro-metastatic vascular niche through Arf6 up regulation. Cancer Microenviron. 7, 41-59. doi: 10.1007/s12307-013-0142-2

Persidsky, Y., Ramirez, S. H., Haorah, J., and Kanmogne, G. D. (2006). Blood-brain barrier: structural components and function under physiologic and pathologic conditions. J. Neuroimmune Pharmacol. 1, 223-236. doi: 10.1007/s11481-0069025-3

Ramirez, S. H., Hasko, J., Skuba, A., Fan, S., Dykstra, H., McCormick, R., et al. (2012). Activation of cannabinoid receptor 2 attenuates leukocyte-endothelial cell interactions and blood-brain barrier dysfunction under inflammatory conditions. J. Neurosci. 32, 4004-4016. doi: 10.1523/JNEUROSCI.4628-11.2012

Raposo, G., and Stoorvogel, W. (2013). Extracellular vesicles: exosomes, microvesicles, and friends. J. Cell Biol. 200, 373-383. doi: 10.1083/jcb. 201211138

Rom, S., Zuluaga-Ramirez, V., Dykstra, H., Reichenbach, N. L., Ramirez, S. H., and Persidsky, Y. (2015). Poly(ADP-ribose) polymerase-1 inhibition in brain endothelium protects the blood-brain barrier under physiologic and neuroinflammatory conditions. J. Cereb. Blood Flow Metab. 35, 28-36. doi: 10.1038/jcbfm.2014.167

Salvador, E., Burek, M., and Förster, C. Y. (2015). Stretch and/or oxygen glucose deprivation (OGD) in an in vitro traumatic brain injury (TBI) model induces calcium alteration and inflammatory cascade. Front. Cell. Neurosci. 9:323. doi: $10.3389 /$ fncel.2015.00323

Salvador, E., Neuhaus, W., and Foerster, C. (2013). Stretch in brain microvascular endothelial cells (cEND) as an in vitro traumatic brain injury model of the blood brain barrier. J. Vis. Exp. e50928. doi: 10.3791/50928

Schaffer, J. L., Rizen, M., L'Italien, G. J., Benbrahim, A., Megerman, J., Gerstenfeld, L. C., et al. (1994). Device for the application of a dynamic biaxially uniform and isotropic strain to a flexible cell culture membrane. J. Orthop. Res. 12, 709-719. doi: 10.1002/jor.1100120514

Shan, R., Szmydynger-Chodobska, J., Warren, O. U., Zink, B. J., Mohammad, F., and Chodobski, A. (2016). A new panel of blood biomarkers for the diagnosis of mild traumatic brain injury/concussion in adults. J. Neurotrauma 33, 49-57. doi: 10.1089/neu.2014.3811

Shlosberg, D., Benifla, M., Kaufer, D., and Friedman, A. (2010). Blood-brain barrier breakdown as a therapeutic target in traumatic brain injury. Nat. Rev. Neurol. 6, 393-403. doi: 10.1038/nrneurol.2010.74

Simard, J. M., Kahle, K. T., and Gerzanich, V. (2010). Molecular mechanisms of microvascular failure in central nervous system injury-synergistic roles of NKCC1 and SUR1/TRPM4. J. Neurosurg. 113, 622-629. doi: 10.3171/2009.11.JNS081052

Tan, K. T., Tayebjee, M. H., Lynd, C., Blann, A. D., and Lip, G. Y. (2005). Platelet microparticles and soluble $\mathrm{P}$ selectin in peripheral artery disease: relationship to extent of disease and platelet activation markers. Ann. Med. 37, 61-66. doi: 10.1080/07853890410018943

Thal, S. C., Luh, C., Schaible, E. V., Timaru-Kast, R., Hedrich, J., Luhmann, H. J., et al. (2012). Volatile anesthetics influence blood-brain barrier integrity by modulation of tight junction protein expression in traumatic brain injury. PLoS ONE 7:e50752. doi: 10.1371/journal.pone.0050752

Unterberg, A. W., Stover, J., Kress, B., and Kiening, K. L. (2004). Edema and brain trauma. Neuroscience 129, 1021-1029. doi: 10.1016/j.neuroscience.2004.06.046

Vion, A. C., Birukova, A. A., Boulanger, C. M., and Birukov, K. G. (2013a). Mechanical forces stimulate endothelial microparticle generation via caspasedependent apoptosis-independent mechanism. Pulm. Circ. 3, 95-99. doi: 10.4103/2045-8932.109921

Vion, A. C., Ramkhelawon, B., Loyer, X., Chironi, G., Devue, C., Loirand, G., et al. (2013b). Shear stress regulates endothelial microparticle release. Circ. Res. 112, 1323-1333. doi: 10.1161/CIRCRESAHA.112.300818

Wang, B. W., Chang, H., Lin, S., Kuan, P., and Shyu, K. G. (2003). Induction of matrix metalloproteinases- 14 and -2 by cyclical mechanical stretch is mediated by tumor necrosis factor-alpha in cultured human umbilical vein endothelial cells. Cardiovasc. Res. 59, 460-469. doi: 10.1016/S0008-6363(03)00428-0

Wen, J., Qian, S., Yang, Q., Deng, L., Mo, Y., and Yu, Y. (2014). Overexpression of netrin-1 increases the expression of tight junction-associated proteins, 
claudin-5, occludin, and ZO-1, following traumatic brain injury in rats. Exp. Ther. Med. 8, 881-886. doi: 10.3892/etm.2014.1818

Whalen, M. J., Carlos, T. M., Dixon, C. E., Schiding, J. K., Clark, R. S., Baum, E., et al. (1999). Effect of traumatic brain injury in mice deficient in intercellular adhesion molecule-1: assessment of histopathologic and functional outcome. J. Neurotrauma 16, 299-309. doi: 10.1089/neu.1999.16.299

Winston, F. K., Macarak, E. J., Gorfien, S. F., and Thibault, L. E. (1989). A system to reproduce and quantify the biomechanical environment of the cell. J. Appl. Physiol. (1985) 67, 397-405.

Conflict of Interest Statement: The authors declare that the research was conducted in the absence of any commercial or financial relationships that could be construed as a potential conflict of interest.
SR is an inventor in a US patent application related to brain endothelial-derived extracellular microvesicles. The US patent application number is filled under US 14/406,400; PCT/US2013/047470 and is entitled "Method for detecting injury to the brain". The application priority date was $6 / 26 / 12$, filling date $6 / 26 / 12$ and publication date $5 / 21 / 2015$.

Copyright $\odot 2016$ Andrews, Lutton, Merkel, Razmpour and Ramirez. This is an open-access article distributed under the terms of the Creative Commons Attribution License (CC BY). The use, distribution or reproduction in other forums is permitted, provided the original author(s) or licensor are credited and that the original publication in this journal is cited, in accordance with accepted academic practice. No use, distribution or reproduction is permitted which does not comply with these terms. 